\title{
FUNCTIONS MONOTONE CLOSE TO BOUNDARY
}

\author{
Olli Martio, Vladimir Miklyukov and Matti VuORinen
}

(Received December 15, 2003, revised December 9, 2004)

\begin{abstract}
Functions which are monotone close to boundary are defined. Some oscillation estimates are given for these functions in Orlicz classes. Criteria for monotonicity close to boundary are obtained.
\end{abstract}

1. Main results. Let $D \subset \boldsymbol{R}^{2}$ be a domain in the Euclidean plane. By $\tilde{\partial} D$ we denote the boundary of $D$ in the extended plane $\tilde{\boldsymbol{R}}^{2}=\boldsymbol{R}^{2} \cup\{\infty\}$. For a subdomain $\Delta \subset D$, we set

$$
\partial^{\prime} \Delta=\tilde{\partial} \Delta \backslash \tilde{\partial} D \quad \text { and } \quad \partial^{\prime \prime} \Delta=\tilde{\partial} \Delta \cap \tilde{\partial} D .
$$

For an arbitrary function $f: D \rightarrow \boldsymbol{R}$ and $A \subset \bar{D}, A \neq \emptyset$, we put

$$
\operatorname{osc}(f, A)=\sup _{a, b \in A} \limsup _{a_{j} \rightarrow a, b_{j} \rightarrow b}\left(f\left(a_{j}\right)-f\left(b_{j}\right)\right),
$$

where the $\lim$ sup is taken over all sequences $a_{j} \rightarrow a, b_{j} \rightarrow b$ of points in $D$. Let $\Gamma$ be a subset of $\tilde{\partial} D$. A continuous function $f: D \rightarrow \boldsymbol{R}$ is called monotone close to $\Gamma$ if for every subdomain $\Delta \subset D$ with $\partial^{\prime \prime} \Delta \subset \Gamma$,

$$
\operatorname{osc}(f, \Delta) \leq \operatorname{osc}\left(f, \partial^{\prime} \Delta\right),
$$

see Martio et al. [10].

If $\Gamma=\emptyset$, then we have the well-known class of monotone functions in the sense of Lebesgue. If $\Gamma=\tilde{\partial} D$, then every function, monotone close to $\Gamma$, is a constant. This is evident, since, choosing $\Delta=D \backslash\left\{x_{0}\right\}$ where $x_{0} \in D$ is an arbitrary point, it follows from (1.1) that

$$
\operatorname{osc}(f, \Delta) \leq \operatorname{osc}\left(f,\left\{x_{0}\right\}\right)=0 .
$$

For another generalization of monotonicity in the sense of Lebesgue, see Manfredi [8].

For $a, b \in D$ we let

$$
\rho_{D}(a, b)=\inf _{\gamma} \operatorname{diam} \gamma
$$

where the infimum is taken over all $\operatorname{arcs} \gamma \subset D$ joining $a$ and $b$. The quantity $\rho_{D}(a, b)$ is called the inner distance between $a$ and $b$. Clearly, $\rho_{D}$ defines a metric in $D$. For arbitrary sets $A, B \subset \boldsymbol{R}^{2}$ we let

$$
\operatorname{dist}(A, B)=\sup _{x \in A} \operatorname{dist}(x, B) .
$$

2000 Mathematics Subject Classification. Primary 31C45; Secondary 46E30.

Key words and phrases. Dirichlet integral, monotone function, oscillation estimate.

The authors are indebted to the anonymous referee for a set of useful remarks. 
For a set $\Gamma \subset \partial D, D \neq \emptyset$, and points $a, b \in D$ we set

$$
\delta_{D}(a, b ; \Gamma)=\lim \sup \operatorname{dist}\left(\gamma_{k}, \partial D \backslash \Gamma\right),
$$

where limsup is taken over all sequences $\left\{\gamma_{k}\right\}$ of arcs $\gamma_{k} \subset D$ joining $a$ and $b$ such that $\operatorname{diam} \gamma_{k} \rightarrow \rho_{D}(a, b)$.

We will employ functions with Sobolev derivatives in some Orlicz classes, see [4, Chapter I]. A function $\Phi: \boldsymbol{R} \rightarrow \boldsymbol{R}_{+}$is called an $N$-function if it admits the representation

$$
\Phi(\tau)=\int_{0}^{|\tau|} p(t) d t,
$$

where $p:(0, \infty) \rightarrow(0, \infty)$ is a positive non-decreasing function, continuous to the right, such that

$$
p(0+)=0, \quad p(\infty)=\lim _{t \rightarrow \infty} p(t)=\infty .
$$

Let $D \subset \boldsymbol{R}^{2}$ be an open set. Let $\Phi$ be an $N$-function. Recall that a function $f: D \rightarrow \boldsymbol{R}$ is $A C L$ (absolutely continuous on lines) if for each cube $Q, \bar{Q} \subset D$, and for $j=1,2$ and for all $z$ in the projection of $Q$ into $\left\{x_{j}=0\right\}$, except a one-dimensional set of measure zero, $t \mapsto f\left(z+t e_{j}\right), z+t e_{j} \in Q$, is absolutely continuous. We say that a continuous function $f: D \rightarrow \boldsymbol{R}$ belongs to $A C L^{\Phi}(D)$ if $f$ is $A C L$ and

$$
\int_{D} \Phi(|\nabla f|) d x_{1} d x_{2}<\infty
$$

Here $\nabla f$ stands for the formal gradient $\left(f_{x_{1}}^{\prime}, f_{x_{2}}^{\prime}\right)$, which exists almost everywhere in $D$. A continuous function $f$ belongs to $A C L^{p}$ if $f \in A C L^{\Phi}$ with $\Phi=t^{p}, p \geq 1$. The class $A C L^{2}$ coincides with the class $B L$ introduced in 1906 by Levi [7] (see also Nikodym [13], Deny and Lions [2], Lelong-Ferrand [6], and Suvorov [14]). For $N$-functions $\Phi$ of the general form, see Miklyukov [11], Kruglikov and Miklyukov [5] and Astala et al. [1]. The boundary behavior of monotone $A C L^{n}$ functions has been studied by these authors and others (see, e.g., Manfredi and Villamor [9] and Mizuta [12] and references therein).

Our main result yields the following inequality for functions monotone close to boundary.

THEOREM 1.4. Let $D$ be a subdomain of $\boldsymbol{R}^{2}, \Gamma \subset \partial D$ an open Jordan arc, and $f \in A C L^{\Phi}(D)$. If $f$ is monotone close to $\Gamma$, then for every pair of points $a, b \in D$ with

$$
\rho_{D}(a, b)<\delta_{D}(a, b ; \Gamma),
$$

the following estimate holds:

$$
|f(a)-f(b)| \leq \kappa_{0}\left(\rho_{D}(a, b) ; \delta_{D}(a, b ; \Gamma), \Phi, I\right),
$$

where

$$
I=\int_{D} \Phi(|\nabla f|) d x_{1} d x_{2},
$$

and $\kappa_{0}$ is the function defined by (3.1). 
Suppose that $D$ is convex. Then $\rho_{D}(a, b)=|b-a|$ and

$$
\delta_{D}(a, b ; \Gamma) \leq 2 \min \{\operatorname{dist}(a, \partial D \backslash \Gamma), \operatorname{dist}(b, \partial D \backslash \Gamma)\}
$$

provided that the condition

$$
\rho_{D}(a, b) \leq \frac{1}{2} \delta_{D}(a, b ; \Gamma)
$$

holds. If $D$ is convex and $\Phi$ satisfies some additional conditions, then the estimate (1.5) implies that $f$ has a continuous extension to $\Gamma$. Specifically, if $D$ is convex, then we suppose that for all $a \geq 1$ and all $\tau>0$,

$$
\Phi(a \tau) \leq c_{\Phi} a^{2} \Phi(\tau)
$$

with some constant $c_{\Phi}$ independent of $a$ and $\tau$, and that

$$
\int_{1}^{\infty} \frac{\Phi(\tau)}{\tau^{3}} d \tau=\infty
$$

COROLlary 1.8. If in the situation of Theorem $1.4, D$ is convex and the $N$-function $\Phi$ satisfies (1.6) and (1.7), then $f$ has a continuous extension to $\Gamma$.

In what follows we give two sufficient conditions for monotonicity close to boundary.

THEOREM 1.9. Let $\Phi$ and $\Psi$ be arbitrary mutually complementary $N$-functions. Let $D \subset \boldsymbol{R}^{2}$ be a subdomain of $\boldsymbol{R}^{2}, \Gamma \subset \partial D$ and $f \in A C L^{\Phi}(D)$. Suppose that there exists a vector field $A(x): D \rightarrow \boldsymbol{R}^{2}$ of the class $L^{\Psi}(D)$ such that for almost all $x \in D$ at which $\nabla f(x) \neq 0$,

$$
\sum_{i=1}^{2} f_{x_{i}}^{\prime}(x) A_{i}(x)>0
$$

If for every subdomain $\Delta \subset D$ such that $\partial^{\prime} \Delta$ is locally rectifiable and $\partial^{\prime \prime} \Delta \subset \Gamma$, and for every $\phi \in A C L^{\Phi}(\Delta) \cap C\left(\Delta \cup \partial^{\prime} \Delta\right)$

$$
\int_{\partial^{\prime} \Delta} \phi\langle A(x), \mathbf{n}\rangle|d x|=\int_{\Delta} \sum_{i=1}^{2} \phi_{x_{i}}^{\prime} A_{i}(x) d x_{1} d x_{2},
$$

then $f$ is monotone close to $\Gamma$.

Because the assumptions in Theorem 1.9 are rather complicated, we illustrate the theorem with an example.

EXAMPLE 1.12. Let $D \subset \boldsymbol{R}^{2}$ be a bounded subdomain with smooth boundary and let $\Gamma \subset \partial D$ be an open proper subarc. Let $f$ be a $C^{1}(D \cup \Gamma)$ solution of the equation

$$
\operatorname{div}\left(|\nabla f|^{p-2} \nabla f\right)=0, \quad p \geq 1 .
$$

Suppose that the normal derivative $\partial f /\left.\partial n\right|_{\Gamma}=0$. If we now choose

$$
A(x)=\nabla f(x)|\nabla f(x)|^{p-2},
$$


then

$$
\sum_{i=1}^{2} f_{x_{i}}^{\prime}(x) A_{i}(x)=|\nabla f|^{p}
$$

and the assumption (1.10) holds. Next, for every function $\phi$ and every subdomain $\Delta$ as in Theorem 1.9, we have

$$
\begin{aligned}
\int_{\partial^{\prime} \Delta} \phi\langle A(x), \mathbf{n}\rangle|d x| & =\int_{\partial \Delta} \phi|\nabla f|^{p-2}\langle\nabla f(x), \mathbf{n}\rangle|d x| \\
& =\int_{\Delta} \phi \operatorname{div}|\nabla f|^{p-2} \nabla f d x_{1} d x_{2}+\int_{\Delta}|\nabla f|^{p-2}\langle\nabla \phi, \nabla f\rangle d x_{1} d x_{2} \\
& =\int_{\Delta} \sum_{i=1}^{2} \phi_{x_{i}}^{\prime} A_{i}(x) d x_{1} d x_{2} .
\end{aligned}
$$

This formula implies (1.11) and by Theorem 1.9, $f$ is monotone close to $\Gamma$.

In order to state the next result, let $\Gamma \subset \partial \tilde{D}$ and let $h: D \rightarrow(0, \infty)$ be a locally Lipschitz function such that $\lim _{x \rightarrow \Gamma} h(x)=0$ and

$$
0<h_{0} \leq \underset{D}{\operatorname{essinf}}|\nabla h(x)| \leq \underset{D}{\operatorname{ess} \sup }|\nabla h(x)| \leq h_{1}<\infty,
$$

where $h_{0}$ and $h_{1}$ are some constants. We let $E_{t}=\{x \in D ; h(x)=t\}$.

THEOREM 1.14. Let $f \in A C L_{\mathrm{loc}}^{p}(D), p>1$, be a bounded function. Suppose that there exists a vector field

$$
A=\left(A_{1}, A_{2}\right): D \rightarrow \boldsymbol{R}^{2}, \quad A \in L^{q}(D), \quad q=p /(p-1)
$$

such that

$$
\begin{aligned}
& \sigma_{1}(x)|\nabla f(x)|^{p} \leq \sum_{i=1}^{2} f_{x_{i}}^{\prime}(x) A_{i}(x), \\
& \left(\sum_{i=1}^{2} A_{i}^{2}(x)\right)^{1 / 2} \leq \sigma_{2}(x)|\nabla f(x)|^{p-1}
\end{aligned}
$$

for some continuous functions $\sigma_{j}: D \rightarrow(0, \infty), j=1,2$, and that (1.11) holds for all functions $\phi \in A C L^{p}(D)$, supp $\phi \subset \subset D$, and for all subdomains $\Delta \subset \subset D$ with locally rectifiable boundaries. If

$$
\int_{0} d t\left(\int_{D \cap E_{t}} \frac{\sigma_{2}^{p}}{\sigma_{1}^{p-1}} d \mathcal{H}^{1}\left(E_{t}\right)\right)^{1 /(1-p)}=\infty,
$$

then $f$ is monotone close to $\Gamma$. In particular, if $\Gamma=\tilde{\partial} D$, then $f \equiv$ constant.

Let $\gamma$ be a simple open Jordan arc lying in the upper half-plane with endpoints $(0,0)$ and $(a, 0)$ on the $x_{1}$-axis. We set

$$
\Gamma=\left\{\left(x_{1}, x_{2}\right) ; 0 \leq x_{1} \leq a, x_{2}=0\right\},
$$


and denote by $D$ the subdomain of $\boldsymbol{R}^{2}$ lying between $\gamma$ and $\Gamma$. Choosing $h(x)=x_{2}$ in Theorem 1.14, we obtain the following result.

COROLLARY 1.17. Let $f$ be as in Theorem 1.14. Suppose that there exists a vector field

$$
A=\left(A_{1}, A_{2}\right): D \rightarrow \boldsymbol{R}^{2}, \quad A \in L^{q}(D), \quad q=p /(p-1),
$$

satisfying

$$
\begin{aligned}
\sigma_{1}\left(x_{2}\right)|\nabla f(x)|^{p} & \leq \sum_{i=1}^{2} f_{x_{i}}^{\prime}(x) A_{i}(x), \\
\left(\sum_{i=1}^{2} A_{i}^{2}(x)\right)^{1 / 2} & \leq \sigma_{2}\left(x_{2}\right)|\nabla f(x)|^{p-1}
\end{aligned}
$$

with some continuous functions $\sigma_{1}, \sigma_{2}>0$. Suppose also that for all functions $\phi \in A C L^{p}(D)$, $\operatorname{supp} \phi \subset \subset D$, and all subdomains $\Delta \subset \subset D$ with locally rectifiable boundaries, the relation (1.11) holds. If

$$
\int_{0} \frac{\sigma_{1}(t)}{\sigma_{2}^{p /(p-1)}(t)} d t=\infty
$$

then $f$ is monotone close to $\Gamma$.

EXAMPLE 1.20. Let $D$ be as in Corollary 1.17 and let $f$ be a $C^{1}$ solution in $D$ of an equation

$$
\operatorname{div}\left(\sigma|\nabla f|^{p-2} \nabla f\right)=0, \quad p>1,
$$

with a continuous weight function $\sigma(x)=\sigma\left(x_{2}\right)$. Setting

$$
A_{i}(x)=\sigma|\nabla f|^{p-2} f_{x_{i}}^{\prime},
$$

we find

$$
\sum_{i=1}^{2} f_{x_{i}}^{\prime} A_{i}=\sigma|\nabla f|^{p} \quad \text { and } \quad|A|=\sigma|\nabla f|^{p-1} .
$$

Thus, (1.18) holds with $\sigma_{1}=\sigma_{2}=\sigma$. The assumption (1.19) takes the form

$$
\int_{0} \sigma^{-1 /(p-1)}(t) d t=\infty
$$

which guarantees that $f$ is monotone close to $\Gamma$.

2. Length and area principle. Each $N$-function is convex. The first condition in (1.3) means that

$$
\lim _{\tau \rightarrow 0} \frac{\Phi(\tau)}{\tau}=0,
$$

and the second condition means that

$$
\lim _{\tau \rightarrow \infty} \frac{\Phi(\tau)}{\tau}=\infty
$$


For every non-decreasing function $p:(0, \infty) \rightarrow(0, \infty)$, which is continuous to the right and satisfies (1.3), we find another function $q:(0, \infty) \rightarrow(0, \infty), s \geq 0$, such that

$$
q(s)=\sup _{p(t) \leq s} t .
$$

It is easy to see that $q(s)$ also satisfies (1.3). The pair of $N$-functions

$$
\Phi(\tau)=\int_{0}^{|\tau|} p(t) d t \quad \text { and } \quad \Psi(\tau)=\int_{0}^{|\tau|} q(s) d s
$$

are called mutually complementary.

We give several estimates for $A C L^{\Phi}$-functions, which are needed for studying functions monotone close to boundary. For this purpose we first recall Orlicz classes. Fix a Lebesgue measurable set $A \subset \boldsymbol{R}^{2}$ and an arbitrary $N$-function $\Phi$. Then the $\operatorname{Orlicz} \operatorname{class} L^{\Phi}(A)$ is defined to be the set of Lebesgue measurable functions $u$ in $A$ such that

$$
I(u, A ; \Phi) \equiv \int_{A} \Phi[u(x)] d x_{1} d x_{2}<\infty .
$$

In applications the functions

$$
\Phi(\tau)=\tau^{2} \ln ^{\alpha}(e+\tau)
$$

play an important role, and the classes $L^{\Phi}(A)$ associated with this particular $\Phi$ are called Zygmund classes [1].

Let $\Phi(\tau)$ and $\Psi(\tau)$ be mutually complementary $N$-functions. By $\tilde{L}^{\Phi}(A)$ we denote the set of the functions $u(x)$ satisfying

Next, we set

$$
\left|\int_{A} u(x) v(x) d x_{1} d x_{2}\right|<\infty \quad \text { for all } v(x) \in L^{\Psi}(A) .
$$

$$
\|u\|_{\Phi, A} \equiv \sup _{I(v, A ; \Psi) \leq 1}\left|\int_{A} u(x) v(x) d x_{1} d x_{2}\right| .
$$

After introducing equivalent norms, the class $\tilde{L}^{\Phi}(A)$ is converted to a linear norm space (see [4, Chapter II]). Namely, the definition (2.3) of the norm implies that:

(1) $\|u\|_{\Phi, A}=0$ if and only if $u(x)=0$ almost everywhere on $A$;

(2) $\|\alpha u\|_{\Phi, A}=|\alpha|\|u\|_{\Phi, A}$;

(3) $\left\|u_{1}+u_{2}\right\|_{\Phi, A} \leq\left\|u_{1}\right\|_{\Phi, A}+\left\|u_{2}\right\|_{\Phi, A}$.

For the next lemma we refer the reader to [4].

LEMMA 2.4. (i) For each $u \in L^{\Phi}(A)$,

$$
\|u\|_{\Phi, A} \leq \int_{A} \Phi(u) d x_{1} d x_{2}+1 .
$$

In particular, $L^{\Phi}(A) \subset \tilde{L}^{\Phi}(A)$.

(ii) If $\|u\|_{\Phi, A} \leq 1$, then $u \in L^{\Phi}(A)$ and

$$
\int_{A} \Phi(u) d x_{1} d x_{2} \leq\|u\|_{\Phi, A} .
$$


In particular,

$$
\int_{A} \Phi\left[\frac{u(x)}{\|u\|_{\Phi, A}}\right] d x_{1} d x_{2} \leq 1 .
$$

(iii) For every pair of functions $u \in \tilde{L}^{\Phi}(A)$ and $v \in \tilde{L}^{\Psi}(A)$,

$$
\left|\int_{A} u(x) v(x) d x_{1} d x_{2}\right| \leq\|u\|_{\Phi, A}\|v\|_{\Psi, A} .
$$

Lemma 2.8. Suppose that a domain $D \subset \boldsymbol{R}^{2}$ is of finite area, $\Phi$ is an $N$-function and $f \in A C L^{\Phi}(D)$. Then $f \in A C L^{1}(D)$.

Proof. Since $\Phi$ satisfies (2.2), there exists $q>0$ such that

$$
\frac{\tau}{\Phi(\tau)} \leq 1 \text { for all } \tau \geq q
$$

Writing

$$
D_{1}=\{x \in D ;|\nabla f(x)| \leq q\}, \quad D_{2}=\{x \in D ;|\nabla f(x)|>q\}
$$

we have

$$
\begin{aligned}
\int_{D}|\nabla f| d x_{1} d x_{2} & =\int_{D_{1}}|\nabla f| d x_{1} d x_{2}+\int_{D_{2}}|\nabla f| d x_{1} d x_{2} \\
& \leq q \int_{D_{1}} d x_{1} d x_{2}+\int_{D_{2}} \frac{|\nabla f|}{\Phi(|\nabla f|)} \Phi(|\nabla f|) d x_{1} d x_{2} \\
& \leq q \mathcal{H}^{2}\left(D_{1}\right)+C \int_{D_{2}} \Phi(|\nabla f|) d x_{1} d x_{2}
\end{aligned}
$$

where $\mathcal{H}^{2}$ is the two-dimensional Lebesgue measure and

$$
C=\sup _{x \in D_{2}} \frac{|\nabla f(x)|}{\Phi(|\nabla f(x)|)} \leq 1 .
$$

Hence, we obtain

$$
\begin{aligned}
\int_{D}|\nabla f| d x_{1} d x_{2} & \leq q \mathcal{H}^{2}\left(D_{1}\right)+\int_{D_{2}} \Phi(|\nabla f|) d x_{1} d x_{2} \\
& \leq q \mathcal{H}^{2}(D)+\int_{D} \Phi(|\nabla f|) d x_{1} d x_{2}
\end{aligned}
$$

and $f$ is $A C L^{1}$ as required.

EXAMPLE 2.9. The functions $\Phi(\tau)=\tau^{2}$ and $\Phi(\tau)=\tau^{2} / \ln (e+|\tau|)$ are typical examples of $N$-functions satisfying (1.6) and (1.7). This is evident for $\Phi(\tau)=\tau^{2}$. We verify the necessary conditions for $\Phi(\tau)=\tau^{2} / \ln (e+|\tau|)$.

First, we observe that this function satisfies (1.3). Indeed, the derivative of $\Phi(\tau)$ has the form

$$
\Phi^{\prime}(\tau)=\tau \frac{2 \ln (e+\tau)-\tau /(e+\tau)}{\ln ^{2}(e+\tau)}, \quad \tau>0
$$


Clearly, $\Phi^{\prime}(0+)=0$. Next, we note that

$$
\ln (e+\tau)>1 \text { for } \tau>0 .
$$

Hence, for all $\tau>0$,

$$
\begin{aligned}
\Phi^{\prime}(\tau) & =\tau \frac{2(e+\tau) \ln (e+\tau)-\tau}{(e+\tau) \ln ^{2}(e+\tau)}>\tau \frac{2(e+\tau)-\tau}{(e+\tau) \ln ^{2}(e+\tau)} \\
& =\tau \frac{2 e+\tau}{(e+\tau) \ln ^{2}(e+\tau)}>0
\end{aligned}
$$

from which we see

$$
\Phi^{\prime}(\infty)=\lim _{\tau \rightarrow \infty} \Phi^{\prime}(\tau)=\infty .
$$

Secondly, we verify (1.6). For every $a \geq 1$ we have

$$
\Phi(a \tau)=a^{2} \tau^{2} / \ln (e+a|\tau|) \leq a^{2} \tau^{2} / \ln (e+|\tau|)=a^{2} \Phi(\tau) .
$$

The property (1.7) is evident.

We denote by $S_{D}(a, r)$ the intersection of the circle $S(a, r)$ and the set $D \subset \boldsymbol{R}^{2}$. Fix $\varepsilon$ and $\varepsilon_{0}$ such that $0<\varepsilon<\varepsilon_{0}<\infty$. Suppose that for all $\tau \in\left(\varepsilon, \varepsilon_{0}\right)$ the intersection $S_{D}(a, \tau) \neq \emptyset$. We set

$$
D_{\varepsilon, \varepsilon_{0}}=\bigcup_{\varepsilon<\tau<\varepsilon_{0}} S_{D}(a, \tau), \quad l(a, r)=\mathcal{H}^{1}\left(S_{D}(a, r)\right) .
$$

Let $\sigma_{1}, \sigma_{2}, \ldots$ be the components of $S_{D}(a, r)$. Then $S_{D}(a, r)=\bigcup_{i} \sigma_{i}$ and for a function $f: D \rightarrow \boldsymbol{R}$ we set

$$
W_{f}(a, r)=\sum_{i} \operatorname{osc}\left(f, \sigma_{i}\right) .
$$

We prove the following version of the Length and Area Principle for $A C L^{\Phi}$-functions. A closely related class of functions was considered in [5].

THEOREM 2.10. Let $\Phi$ be an $N$-function. Then for all $f \in A C L^{\Phi}(D)$ and all $0<$ $\varepsilon<\varepsilon_{0}<\infty$,

$$
\int_{\varepsilon}^{\varepsilon_{0}} \Phi\left(\frac{W_{f}(a, \tau)}{l(a, \tau)}\right) l(a, \tau) d \tau \leq I\left(D_{\varepsilon, \varepsilon_{0}}\right)
$$

where

$$
I\left(D_{\varepsilon, \varepsilon_{0}}\right)=\int_{D_{\varepsilon, \varepsilon_{0}}} \Phi(|\nabla f|) d x_{1} d x_{2} .
$$

ProOF. We may assume that $a=0$. Lemma 2.8 shows that a function $f \in A C L^{\Phi}$ belongs to $A C L_{\text {loc }}^{1}(D)$. Let $(r, \theta)$ be polar coordinates in $\boldsymbol{R}^{2}$ and set $f^{*}=f(r, \theta)$. Now $f^{*} \in$ $A C L_{\mathrm{loc}}^{1}(D)$ and, in particular, $f^{*}$ is absolutely continuous on each line segment $\alpha \leq \theta \leq \beta$ on $S_{D}(0, \tau)$ for almost all $\tau$. Hence, for almost all $\tau \in\left(\varepsilon, \varepsilon_{0}\right)$, we have

$$
W_{f}(0, \tau)=\sum_{i} \operatorname{osc}\left(f, \sigma_{i}\right) \leq \sum_{i} \int_{\sigma_{i}}|\nabla f||d x| \leq \int_{S_{D}(0, \tau)}|\nabla f||d x| .
$$


Recall that $\sigma_{i}$ are the components of $S_{D}(0, \tau)$.

Jensen's integral inequality yields

$$
\Phi\left(\frac{\int_{E} a(x) d \mathcal{H}^{1}}{\mathcal{H}^{1}(E)}\right) \leq \frac{1}{\mathcal{H}^{1}(E)} \int_{E} \Phi(a(x)) d \mathcal{H}^{1}
$$

for an arbitrary $N$-function $\Phi$ and every integrable function $a$ [4, (8.2)]. Using (2.12) for almost all $\tau \in\left(\varepsilon, \varepsilon_{0}\right)$, we obtain

$$
\Phi\left(\frac{W_{f}(0, \tau)}{l(0, \tau)}\right) \leq \Phi\left(\frac{1}{l(0, \tau)} \int_{S_{D}(0, \tau)}|\nabla f||d x|\right) \leq \frac{1}{l(0, \tau)} \int_{S_{D}(0, \tau)} \Phi(|\nabla f|)|d x| .
$$

The functions on both sides of this inequality are measurable with respect to $\tau \in\left(\varepsilon, \varepsilon_{0}\right)$. Multiplying this inequality by $l(0, \tau)$ and integrating over $\left(\varepsilon, \varepsilon_{0}\right)$, we obtain

$$
\int_{\varepsilon}^{\varepsilon_{0}} \Phi\left(\frac{W_{f}(0, \tau)}{l(0, \tau)}\right) l(0, \tau) d \tau \leq \int_{\varepsilon}^{\varepsilon_{0}} d \tau \int_{S_{D}(0, \tau)} \Phi(|\nabla f|)|d x|=\int_{D_{\varepsilon, \varepsilon_{0}}} \Phi(|\nabla f|) d x_{1} d x_{2} .
$$

The following might be the most useful corollary of Theorem 2.10.

COROLlary 2.13. For every $N$-function $\Phi$ and $f \in A C L^{\Phi}(D)$,

$$
\int_{\varepsilon}^{\varepsilon_{0}} \Phi\left(\frac{W_{f}(a, \tau)}{2 \pi \tau}\right) \tau d \tau \leq \frac{1}{2 \pi} I\left(D_{\varepsilon, \varepsilon_{0}}\right) .
$$

Proof. Every $N$-function $\Phi$ satisfies

$$
\Phi(\alpha \tau) \leq \alpha \Phi(\tau)
$$

for all $\tau \geq 0$ and $\alpha \in[0,1]$. Indeed, write $\Phi$ in the form $\Phi(\tau)=\int_{0}^{\tau} p(t) d t$ as in (1.2). Then

$$
\alpha \Phi(\tau)=\int_{0}^{\tau} p(t) d(\alpha t) \geq \int_{0}^{\tau} p(\alpha t) d(\alpha t)=\int_{0}^{\alpha \tau} p(t) d t=\Phi(\alpha \tau)
$$

as required. Let $W \geq 0$. From (2.15) we conclude that

$$
\Phi\left(\frac{W}{2 \pi \tau}\right)=\Phi\left(\frac{W}{l(a, \tau)} \frac{l(a, \tau)}{2 \pi \tau}\right) \leq \Phi\left(\frac{W}{l(a, \tau)}\right) \frac{l(a, \tau)}{2 \pi \tau} .
$$

Hence,

$$
\Phi\left(\frac{W_{f}(a, \tau)}{2 \pi \tau}\right) 2 \pi \tau \leq \Phi\left(\frac{W_{f}(a, \tau)}{l(a, \tau)}\right) l(a, \tau),
$$

and the desired conclusion follows from (2.11).

3. The function $k_{0}$. Here we study the inequality (2.14). For an $N$-function $\Phi$ and an interval $\left(\varepsilon, \varepsilon_{0}\right)$ with $0<\varepsilon<\varepsilon_{0}$, we set

$$
\kappa_{0}\left(\varepsilon ; \varepsilon_{0}, \Phi, I\right)=\sup \left\{\kappa ; \int_{\varepsilon}^{\varepsilon_{0}} \Phi\left(\frac{\kappa}{2 \pi \tau}\right) \tau d \tau \leq \frac{1}{2 \pi} I\right\},
$$

where

$$
I=\int_{D} \Phi(|\nabla f|) d x_{1} d x_{2}
$$


It follows from (2.1) and (2.2) that the function

$$
F(\kappa)=\int_{\varepsilon}^{\varepsilon_{0}} \Phi\left(\frac{\kappa}{2 \pi \tau}\right) \tau d \tau
$$

is continuous and strictly monotone. Moreover,

$$
F(0)=0 \quad \text { and } \quad F(\infty)=\lim _{\kappa \rightarrow \infty} F(\kappa)=\infty .
$$

Now $\kappa_{0}(\varepsilon)=\kappa_{0}\left(\varepsilon ; \varepsilon_{0}, \Phi, I\right)$ is the unique positive root of the equation

$$
F(\kappa)=(I /(2 \pi)) .
$$

The function $F(\kappa)$ has the following properties.

(i) For every $N$-function $\Phi$ the function $\kappa_{0}(\varepsilon)=\kappa_{0}\left(\varepsilon ; \varepsilon_{0}, \Phi, I\right)$ is continuous and strictly increasing on $\left(0, \varepsilon_{0}\right)$. Moreover, $\kappa_{0}$ depends continuously on $\varepsilon_{0}$.

The proof is evident.

(ii) If an $N$-function $\Phi$ satisfies (1.7), then $\lim _{\varepsilon \rightarrow 0} \kappa_{0}(\varepsilon)=0$.

Indeed, suppose that there exists a number $m>0$ such that for all sufficiently small $\varepsilon>0$

$$
\kappa_{0}(\varepsilon) \geq m>0 .
$$

Then by the definition (3.2) of $\kappa_{0}(\varepsilon)$ and the monotonicity of $\Phi$, we have

$$
\int_{\varepsilon}^{\varepsilon_{0}} \Phi\left(\frac{m}{2 \pi \tau}\right) \tau d \tau \leq \frac{1}{2 \pi} I .
$$

Substituting $t=m / 2 \pi \tau$ in this inequality, we find

$$
\left(\frac{m}{2 \pi}\right)^{2} \int_{m / 2 \pi \varepsilon_{0}}^{m / 2 \pi \varepsilon} \frac{\Phi(t)}{t^{3}} d t \leq \frac{1}{2 \pi} I \text { for all } 0<\varepsilon<\varepsilon_{0} .
$$

Letting $\varepsilon \rightarrow 0+$ we obtain a contradiction to (1.7).

(iii) If an $N$-function $\Phi$ satisfies (1.6), then

$$
\lim \inf _{\varepsilon \rightarrow 0+} \kappa_{0}(\varepsilon) /\left(\ln \frac{1}{\varepsilon}\right)^{-\beta}=\infty \quad \text { for every } \beta>1 .
$$

Indeed, suppose that this is not true; that is, for some sequence $\varepsilon_{i} \rightarrow 0$ of positive numbers the inequality

$$
\kappa_{0}\left(\varepsilon_{i}\right) \leq c\left(\ln \frac{1}{\varepsilon_{i}}\right)^{-\beta}
$$

holds with some constant $c>0$. From (3.2) we obtain

$$
\int_{\varepsilon_{i}}^{\varepsilon_{0}} \Phi\left(\frac{\kappa_{0}\left(\varepsilon_{i}\right)}{2 \pi \tau}\right) \tau d \tau=\frac{1}{2 \pi} I
$$

and hence

$$
\int_{\varepsilon_{i}}^{\varepsilon_{0}} \Phi\left(\frac{c}{2 \pi \tau}\left(\ln \frac{1}{\varepsilon_{i}}\right)^{-\beta}\right) \tau d \tau \geq \frac{1}{2 \pi} I
$$


When $\varepsilon_{0} \leq 1$, from (1.6) and (2.15) we have

$$
\begin{aligned}
\frac{1}{2 \pi} I & \leq \int_{\varepsilon_{i}}^{\varepsilon_{0}} \Phi\left(\frac{c}{2 \pi \tau}\left(\ln \frac{1}{\varepsilon_{i}}\right)^{-\beta}\right) \tau d \tau \leq c_{\Phi} \Phi\left(\frac{c}{2 \pi}\left(\ln \frac{1}{\varepsilon_{i}}\right)^{-\beta}\right) \int_{\varepsilon_{i}}^{\varepsilon_{0}} \frac{d \tau}{\tau} d \tau \\
& \leq c_{\Phi}\left(\ln \frac{1}{\varepsilon_{i}}\right)^{-\beta} \Phi\left(\frac{c}{2 \pi}\right) \ln \frac{1}{\varepsilon_{i}} \leq c_{\Phi}\left(\ln \frac{1}{\varepsilon_{i}}\right)^{1-\beta} \Phi\left(\frac{c}{2 \pi}\right) .
\end{aligned}
$$

When $\varepsilon_{0}>1$, we also have

$$
\begin{aligned}
\frac{1}{2 \pi} I & \leq \int_{\varepsilon_{i}}^{1} \Phi\left(\frac{c}{2 \pi \tau}\left(\ln \frac{1}{\varepsilon_{i}}\right)^{-\beta}\right) \tau d \tau+\int_{1}^{\varepsilon_{0}} \Phi\left(\frac{c}{2 \pi \tau}\left(\ln \frac{1}{\varepsilon_{i}}\right)^{-\beta}\right) \tau d \tau \\
& \leq c_{\Phi} \Phi\left(\frac{c}{2 \pi}\right)\left(\ln \frac{1}{\varepsilon_{i}}\right)^{1-\beta}+\left(\ln \frac{1}{\varepsilon_{i}}\right)^{-\beta} \int_{1}^{\varepsilon_{0}} \Phi\left(\frac{c}{2 \pi \tau}\right) \tau d \tau .
\end{aligned}
$$

For $\beta>1$ we obtain a contradiction as $i \rightarrow \infty$.

(iv) We assume here that an $N$-function $\Phi$ satisfies (1.6) and (1.7). Then it is possible to find a majorant for $\kappa_{0}(\varepsilon)$. For this fix $0<\varepsilon<\varepsilon_{0}$ and set $\kappa_{0}=\kappa_{0}(\varepsilon)$. Substituting $t=\kappa_{0} / 2 \pi \tau$ in (3.3), we have

$$
2 \pi I=\kappa_{0}^{2} \int_{\kappa_{0} / 2 \pi \varepsilon_{0}}^{\kappa_{0} / 2 \pi \varepsilon} \frac{\Phi(t)}{t^{3}} d t .
$$

By property (ii) there exists a number $\alpha_{1}=\alpha_{1}\left(\varepsilon_{0}\right)$ such that

$$
\kappa_{0}(\varepsilon) \leq 2 \pi \varepsilon_{0} \text { for all } 0<\varepsilon<\alpha_{1},
$$

and hence for all sufficiently small $\varepsilon>0$,

$$
2 \pi I \geq \kappa_{0}^{2} \int_{1}^{\kappa_{0} / 2 \pi \varepsilon} \frac{\Phi(t)}{t^{3}} d t
$$

By (iii), we see that

$$
\liminf _{\varepsilon \rightarrow 0+} \frac{\kappa_{0}(\varepsilon)}{\sqrt{\varepsilon}}=\infty
$$

and hence there exists a number $\alpha_{2}=\alpha_{2}\left(\varepsilon_{0}\right)$ such that

$$
\kappa_{0}(\varepsilon) \geq 2 \pi \sqrt{\varepsilon} \text { for all } 0<\varepsilon<\alpha_{2} .
$$

Therefore, for all sufficiently small $\varepsilon>0$, we find

$$
2 \pi I \geq \kappa_{0}^{2} \int_{1}^{1 / \sqrt{\varepsilon}} \frac{\Phi(t)}{t^{3}} d t,
$$

and arrive at the estimate

$$
\kappa_{0}\left(\varepsilon, \varepsilon_{0} ; \Phi, I\right) \leq\left(2 \pi I / \int_{1}^{1 / \sqrt{\varepsilon}} \frac{\Phi(t)}{t^{3}} d t\right)^{1 / 2},
$$

which holds for all $0<\varepsilon<\min \left\{\alpha_{1}\left(\varepsilon_{0}\right), \alpha_{2}\left(\varepsilon_{0}\right)\right\}$.

From (3.4) we obtain the following. 
REMARK 3.5. For the $N$-functions $\Phi$ satisfying (1.6) and (1.7), and for all $\varepsilon>0$ with $0<\varepsilon<\min \left\{\alpha_{1}\left(\varepsilon_{0}\right), \alpha_{2}\left(\varepsilon_{0}\right)\right\}$, the inequality (2.14) can be written in the form

$$
\inf _{\varepsilon<\tau<\varepsilon_{0}} W_{f}(a, \tau) \leq\left(2 \pi I / \int_{1}^{1 / \sqrt{\varepsilon}} \frac{\Phi(t)}{t^{3}} d t\right)^{1 / 2},
$$

where

$$
I=\int_{D} \Phi(|\nabla f|) d x_{1} d x_{2}
$$

4. Proof of Theorem 1.4. Fix a number $h>0$ such that

$$
\rho_{D}(a, b)+h<\delta_{D}(a, b ; \Gamma)-h,
$$

and choose an $\operatorname{arc} \gamma \subset D$ connecting $a$ and $b$ with

$$
\operatorname{diam} \gamma<\rho_{D}(a, b)+h .
$$

Let $x_{0} \in \gamma$ and consider a family of circles $\left\{S\left(x_{0}, \tau\right)\right\}$, where $\tau \in\left[\varepsilon, \varepsilon_{0}\right]$ and

$$
\varepsilon=\rho_{D}(a, b)+h, \quad \varepsilon_{0}=\delta_{D}(a, b ; \Gamma)-h .
$$

By Corollary 2.13 and definition (3.1) we obtain

$$
\inf _{\tau \in\left(\varepsilon, \varepsilon_{0}\right)} W_{f}\left(x_{0}, \tau\right) \leq \kappa_{0}\left(\varepsilon ; \varepsilon_{0}, \Phi, I\right) .
$$

For every $\tau \in\left[\varepsilon, \varepsilon_{0}\right]$, each circle $S\left(x_{0}, \tau\right)$ separates points $a, b$ from $\partial D \backslash \Gamma$. Hence, we can find a component $\sigma$ of $S_{D}\left(x_{0}, \tau\right)$ separating $a, b$ from $\partial D \backslash \Gamma$. Because $\tau \in\left[\varepsilon, \varepsilon_{0}\right]$, the ends of $\sigma$ lie on $\Gamma$. We denote by $D_{\sigma}$ the set of the points which are separated by $\sigma$ from $\partial D \backslash \Gamma$.

The function $f$ is monotone close to $\Gamma$ and therefore,

$$
\operatorname{osc}\left(f, D_{\sigma}\right) \leq \operatorname{osc}\left(f, \partial^{\prime} D_{\sigma}\right) .
$$

Hence we have

$$
\begin{aligned}
|f(a)-f(b)| & \leq \inf _{\tau \in\left(\varepsilon, \varepsilon_{0}\right)} \operatorname{osc}\left(f, D_{\sigma}\right) \leq \inf _{\tau \in\left(\varepsilon, \varepsilon_{0}\right)} \operatorname{osc}\left(f, \partial^{\prime} D_{\sigma}\right) \\
& \leq \inf _{\tau \in\left(\varepsilon, \varepsilon_{0}\right)} W_{f}\left(x_{0}, \tau\right) \leq \kappa_{0}\left(\varepsilon ; \varepsilon_{0}, \Phi, I\right) \\
& \leq \kappa_{0}\left(\rho_{D}(a, b)+h ; \delta_{D}(a, b ; \Gamma)-h, \Phi, I\right) .
\end{aligned}
$$

Letting $h \rightarrow 0$, we obtain (1.5).

By (1.7) and by property (ii) in Section 3 , we see that $\kappa_{0}\left(\rho_{D}(a, b)\right) \rightarrow 0$ as $\rho_{D}(a, b) \rightarrow$ 0 . This means that $f$ can be continuously extended to $\Gamma$.

5. Proof of Theorem 1.9. Fix a subdomain $\Delta$ of $D$ with $\partial^{\prime \prime} \Delta \subset \Gamma$. First we prove that

$$
\sup _{\Delta} f(x)=\sup _{\partial^{\prime} \Delta} f(x) \text {. }
$$

Suppose that this is not the case, that is, there exists a point $x_{0} \in \Delta$ such that

$$
f\left(x_{0}\right)>\sup _{\partial^{\prime} \Delta} f(x)=M .
$$


Choose $\varepsilon>M$ such that $f\left(x_{0}\right)>\varepsilon$. By Lemma 2.8 the function $f \in A C L^{1}(D)$ and, by [15, Theorem 5.4.4], for almost all $\varepsilon$ the set $\{x \in \Delta ; f(x)=\varepsilon\}$ is locally rectifiable. Fix a component $U, x_{0} \in U$, of the set $\{x \in \Delta ; f(x)>\varepsilon\}$. Without loss of generality, we may assume that $\partial^{\prime} U$ is locally rectifiable. Using (1.11) with $\phi=f(x)-\varepsilon$, we write

$$
\int_{U} \sum_{i=1}^{2} f_{x_{i}}^{\prime} A_{i}(x) d x_{1} d x_{2}=\int_{\partial^{\prime} U}(f-\varepsilon)\langle A(x), \mathbf{n}\rangle|d x|=0 .
$$

(Since $\Phi$ and $\Psi$ are mutually complementary, it follows from (2.5) and (2.7) that the left-hand integral and, hence, the right-hand integral exist.) From (1.10) it follows that

$$
\nabla f(x)=0 \quad \text { almost everywhere on } U,
$$

and $f \equiv$ constant on $U$, which leads to a contradiction with the definition of the component $U, x_{0} \in U$. Thus (5.1) follows.

Since $-f$ also satisfies (1.10), (5.1) yields

$$
\inf _{\Delta} f(x)=\inf _{\partial^{\prime} \Delta} f(x) .
$$

Finally, (5.1) and (5.2) imply (1.1).

6. Proof of Theorem 1.14. Fix a subdomain $\Delta$ of $D$ with $\partial^{\prime \prime} \Delta \subset \Gamma$. As in the proof of Theorem 1.9, it suffices to prove (5.1). Suppose that (5.1) is not true; that is, there is $x_{0} \in \Delta$ such that

$$
f\left(x_{0}\right)>M_{0} \equiv \sup _{\partial^{\prime} \Delta} f(x) .
$$

As above, for some $\varepsilon, f\left(x_{0}\right)>\varepsilon>M_{0}$, we choose a component $U$ of $\{x \in \Delta ; f(x)>\varepsilon\}$ with a locally rectifiable boundary $\partial U$ along which $f(x)-\varepsilon=0$.

Fix numbers $0<\delta^{\prime}<\delta^{\prime \prime}<h\left(x_{0}\right)$ and a non-negative Lipschitz function $\psi_{0}: \boldsymbol{R}_{+} \rightarrow \boldsymbol{R}$. Define $\psi: \boldsymbol{R}_{+} \rightarrow \boldsymbol{R}$ by

$$
\psi(\tau)= \begin{cases}1 & \text { for } \delta^{\prime \prime}<\tau<\infty \\ \psi_{0}(\tau) & \text { for } \delta^{\prime} \leq \tau \leq \delta^{\prime \prime} \\ 0 & \text { for } 0<\tau<\delta^{\prime}\end{cases}
$$

Denote $\phi=\psi^{p}(f-\varepsilon)$ with $\psi=\psi(h(x))$ for $x \in U$ and $\phi \equiv 0$ for $x \in D \backslash U$. Clearly, $\phi \in A C L^{p}(D)$ and $\operatorname{supp} \phi \subset \subset D$. Applying (1.11) to $\phi$, we have

$$
\int_{\partial^{\prime} U} \psi^{p}(f-\varepsilon)\langle A, \mathbf{n}\rangle|d x|=\int_{U} \psi^{p}\langle\nabla f, A\rangle d x_{1} d x_{2}+p \int_{U} \psi^{p-1}(f-\varepsilon)\langle\nabla \psi, A\rangle d x_{1} d x_{2} .
$$

Since the contour integral vanishes, we see that

$$
\begin{aligned}
\int_{U} \psi^{p}\langle\nabla f, A\rangle d x_{1} d x_{2} & =-p \int_{U} \psi^{p-1}(f-\varepsilon)\langle\nabla \psi, A\rangle d x_{1} d x_{2} \\
& \leq p \int_{U} \psi^{p-1}|f-\varepsilon||\nabla \psi \| A| d x_{1} d x_{2}
\end{aligned}
$$


Using (1.15), we then obtain

$$
\begin{aligned}
\int_{U} \psi^{p} \sigma_{1}|\nabla f|^{p} d x_{1} d x_{2} \\
\quad \leq p \int_{U} \psi^{p-1}|f-\varepsilon||\nabla \psi| \sigma_{2}|\nabla f|^{p-1} d x_{1} d x_{2} \\
\quad \leq p M \int_{U} \psi^{p-1} \frac{\sigma_{1}^{(p-1) / p}}{\sigma_{1}^{(p-1) / p}}|\nabla \psi| \sigma_{2}|\nabla f|^{p-1} d x_{1} d x_{2} \\
\quad \leq p M\left(\int_{U} \frac{\sigma_{2}^{p}}{\sigma_{1}^{p-1}}|\nabla \psi|^{p} d x_{1} d x_{2}\right)^{1 / p}\left(\int_{U} \psi^{p} \sigma_{1}|\nabla f|^{p} d x_{1} d x_{2}\right)^{(p-1) / p}
\end{aligned}
$$

where

and hence

$$
M=\sup _{x \in U}|f(x)-\varepsilon|,
$$

$$
\int_{U} \psi^{p} \sigma_{1}|\nabla f|^{p} d x_{1} d x_{2} \leq p^{p} M^{p} \int_{U} \frac{\sigma_{2}^{p}}{\sigma_{1}^{p-1}}|\nabla \psi|^{p} d x_{1} d x_{2}
$$

Let

$$
U\left(\delta^{\prime \prime}\right)=\left\{x \in U ; \delta^{\prime \prime}<h(x)\right\}, \quad U\left(\delta^{\prime}, \delta^{\prime \prime}\right)=\left\{x \in U ; \delta^{\prime}<h(x)<\delta^{\prime \prime}\right\} .
$$

Since $\delta^{\prime \prime}<h\left(x_{0}\right)$ and $x_{0} \in U$, the set $U\left(\delta^{\prime \prime}\right) \neq \varnothing$. Noting the specific structure of $\psi$ and using (6.1), we arrive at the inequality

$$
\int_{U\left(\delta^{\prime \prime}\right)} \sigma_{1}|\nabla f|^{p} d x_{1} d x_{2} \leq p^{p} M^{p} \int_{U\left(\delta^{\prime}, \delta^{\prime \prime}\right)} \frac{\sigma_{2}^{p}}{\sigma_{1}^{p-1}}\left|\nabla \psi_{0}\right|^{p} d x_{1} d x_{2} .
$$

We have $\left|\nabla \psi_{0}\right|=\left|\psi_{0}^{\prime}\right||\nabla h|$. By (1.13) together with the well-known co-area formula [3, Section 3.2], we find that

$$
\int_{U\left(\delta^{\prime}, \delta^{\prime \prime}\right)} \frac{\sigma_{2}^{p}}{\sigma_{1}^{p-1}}\left|\nabla \psi_{0}\right|^{p} d x_{1} d x_{2}=\int_{\delta^{\prime}}^{\delta^{\prime \prime}}\left|\psi_{0}^{\prime}(\tau)\right|^{p} d \tau \int_{U \cap E_{\tau}} \frac{\sigma_{2}^{p}}{\sigma_{1}^{p-1}}|\nabla h|^{p-1} d \mathcal{H}^{1}\left(E_{\tau}\right),
$$

and then

$$
\int_{U\left(\delta^{\prime}, \delta^{\prime \prime}\right)} \frac{\sigma_{2}^{p}}{\sigma_{1}^{p-1}}\left|\nabla \psi_{0}\right|^{p} d x_{1} d x_{2} \leq h_{1}^{p-1} \int_{\delta^{\prime}}^{\delta^{\prime \prime}}\left|\psi_{0}^{\prime}(\tau)\right|^{p} d \tau \int_{U \cap E_{\tau}} \frac{\sigma_{2}^{p}}{\sigma_{1}^{p-1}} d \mathcal{H}^{1}\left(E_{\tau}\right) .
$$

Thus we obtain

$$
\int_{U\left(\delta^{\prime \prime}\right)} \sigma_{1}|\nabla f|^{p} d x_{1} d x_{2} \leq p^{p} M^{p} h_{1}^{p-1} \int_{\delta^{\prime}}^{\delta^{\prime \prime}} \xi(\tau)\left|\psi_{0}^{\prime}(\tau)\right|^{p} d \tau,
$$

where

$$
\xi(\tau)=\int_{E_{\tau}} \frac{\sigma_{2}^{p}}{\sigma_{1}^{p-1}} d \mathcal{H}^{1}\left(E_{\tau}\right) .
$$


We choose

$$
\psi_{0}(\tau)=\int_{\delta^{\prime}}^{\tau} \xi^{1 /(1-p)}(t) / \int_{\delta^{\prime}}^{\delta^{\prime \prime}} \xi^{1 /(1-p)}(t) d t \quad \text { for } \delta^{\prime} \leq \tau \leq \delta^{\prime \prime} .
$$

Then

and from (6.2) we have

$$
\int_{\delta^{\prime}}^{\delta^{\prime \prime}} \xi(\tau)\left|\psi_{0}^{\prime}(\tau)\right|^{p} d \tau=\left(\int_{\delta^{\prime}}^{\delta^{\prime \prime}} \xi^{1 /(1-p)}(\tau) d \tau\right)^{1-p}
$$

$$
\int_{U\left(\delta^{\prime \prime}\right)} \sigma_{1}|\nabla f|^{p} d x_{1} d x_{2} \leq p^{p} M^{p} h_{1}^{p-1}\left(\int_{\delta^{\prime}}^{\delta^{\prime \prime}} \xi^{1 /(1-p)}(\tau) d \tau\right)^{1-p}
$$

which holds for every $0<\delta^{\prime}<\delta^{\prime \prime}$. Letting $\delta^{\prime} \rightarrow 0+$ and using (1.16), we obtain

$$
\int_{U\left(\delta^{\prime \prime}\right)} \sigma_{1}|\nabla f|^{p} d x_{1} d x_{2}=0
$$

and, in particular, $\nabla f \equiv 0$ on $U\left(\delta^{\prime \prime}\right)$. Since $\delta^{\prime \prime}<h\left(x_{0}\right)$ is arbitrary, we see that $\nabla f \equiv 0$ on $U$, which means that $f \equiv \varepsilon$ on $U$. This is again a contradiction to the definition of $U$ and the rest of the proof proceeds as in the end of the proof of Theorem 1.9.

7. Two examples. Let $D \subset \boldsymbol{R}^{2}$ be a domain with a Jordan boundary $\partial D$. Let $\Gamma \subset \partial D$ be an open arc. Let $f: \bar{D} \rightarrow \boldsymbol{R}$ be a continuous function, monotone in the sense of Lebesgue in $D$, such that the restriction $\left.f\right|_{\Gamma}$ has no points of strict local extremum ${ }^{1}$. Then $f$ is monotone close to $\Gamma$.

For the proof let $\Delta$ be a subdomain of $D$ with $\partial^{\prime \prime} \Delta \subset \Gamma$. The function $f$, being monotone in the sense of Lebesgue, takes its maximum and minimum values in $\partial \Delta$. Hence there exist $x_{1}, x_{2} \in \partial \Delta$ such that

$$
\sup _{x \in \Delta} f(x)=f\left(x_{1}\right), \quad \inf _{x \in \Delta} f(x)=f\left(x_{2}\right) .
$$

If $x_{1} \notin \Gamma$, then

$$
\sup _{x \in \Delta} f(x)=\sup _{x \in \partial^{\prime} \Delta} f(x) \text {. }
$$

For $x_{1} \in \Gamma$ there are two possibilities (a) $x_{1} \in \operatorname{Clo}\left(\partial^{\prime} \Delta\right)$ and (b) $x_{1} \notin \operatorname{Clo}\left(\partial^{\prime} \Delta\right)$. By continuity, (7.1) holds in case (a). In case (b), there is an open neighborhood $U$ of $x_{1}$ on $\Gamma$ such that $U \subset \partial^{\prime \prime} \Delta$. Since $f$ does not have a strict maximum on $\Gamma, f$ takes greater values on $\partial \Delta$ than $f\left(x_{1}\right)$. This is a contradiction. Hence (7.1) always holds. The point $x_{2}$ can be handled similarly. Thus, we obtain

$$
\operatorname{osc}(f, \Delta)=f\left(x_{1}\right)-f\left(x_{2}\right)=\sup _{x \in \partial^{\prime} \Delta} f(x)-\inf _{x \in \partial^{\prime} \Delta} f(x)=\operatorname{osc}\left(f, \partial^{\prime} \Delta\right) .
$$

Consequently, the function $f$ is monotone close to $\Gamma$.

There exist non-constant functions, monotone close to boundary, which do not have con-

\footnotetext{
${ }^{1}$ A continuous function $u: \Gamma \rightarrow \boldsymbol{R}$ has a strict local minimum (maximum) at a point $a \in \Gamma$ if there exists $\varepsilon>0$ such that $u(a)<u(x)(u(a)>u(x))$ for all $x \in \Gamma, 0<|x-a|<\varepsilon$.
} 
tinuous extensions to the boundary.

Let $D=\left\{\left(x_{1}, x_{2}\right) ; x_{2}>0\right\}$ be the upper half-plane. Consider the function

$$
f\left(x_{1}, x_{2}\right)=\sin \frac{1}{x_{2}} .
$$

Clearly, $f$ is monotone close to boundary $\Gamma=\left\{x=\left(x_{1}, x_{2}\right) ; x_{2}=0\right\}$, but it does not have a continuous extension to $\Gamma$.

\section{REFERENCES}

[1] K. Astala, T. Iwaniec, P. Koskela and G. Martin, Mappings of $B M O$-bounded distortion, Math. Ann. 317 (2000), 703-726.

[2] J. Deny And J. L. Lions, Les espaces du type de Beppo Levi, Ann. Inst. Fourier (Grenoble) 5 (1953/54), 305-370.

[ 3 ] H. Federer, Geometric measure theory, Grundlehren Math. Wiss. 153, Springer, New York, 1969.

[ 4 ] M. A. Krasnoselskil and Ya. B. Rutickit, Convex functions and Orlicz spaces, Problems of Contemporary Mathematics, Gosudarstv. Izdat. Fiz.-Mat. Lit., Moscow (in Russian), 1958.

[5] V. I. Kruglikov and V. M. Miklyukov, Certain classes of plane topological mappings with generalized derivatives. Metric questions of the theory of functions and mappings IV, 102-122, (Russian) Izdat. "Naukova Dumka", Kiev, 1973.

[ 6 ] J. LELONG-FERRAND, Représentation conforme et transformations à intégrale de Dirichlet bornée, GauthierVillars, Paris, 1955.

[ 7 ] B. Levi, Sul principio di Dirichlet, Rend. Circ. Mat. Palermo (2) 22 (1906), 293-359.

[ 8 ] J. J. MANFREDI, Weakly monotone functions, J. Geom. Anal. 4 (1994), 393-402.

[9] J. J. MANFREdi AND E. Villamor, Traces of monotone functions in weighted Sobolev functions, Illinois J. Math. 45 (2001), 403-422.

[10] O. Martio, V. Miklyukov and M. Vuorinen, Some remarks on an existence problem for degenerate elliptic systems, Proc. Amer. Math. Soc. 133 (2005), 1451-1458.

[11] V. M. MikLYUKov, Certain classes of mappings on the plane, Dokl. Akad. Nauk SSSR 183 (1968), 772-774. (English translation Soviet Math. Dokl. 9 (1968), 1455-1457.)

[12] Y. MizUTA, Tangential limits of monotone Sobolev functions, Ann. Acad. Sci. Fenn. Math. 20 (1995), 315326.

[13] O. Nikodym, Sur une class de fonctions considérées dans le problem de Dirichlet, Fund. Math. 21 (1933), 129-150.

[14] G. D. Suvorov, The generalized "length and area principle" in mapping theory, "Naukova Dumka", Kiev (in Russian), 1985

[15] W. P. ZIEMER, Weakly differentiable functions, Grad. Texts in Math. 120, Springer, New York, 1989.

Department of Mathematics and Statistics

P.O. BOX 68, FIN-00014

UNIVERSITY OF HELSINKI

FINLAND

E-mail address:martio@cc.helsinki.fi
Department of MATHEMATICS VOLGOGRAD STATE UNIVERSITY UL. 2 PRodol'naya 30

VOLGOGRAD 400062

RUSSIA

E-mail address: miklyuk@vlink.ru 
DEPARTMENT OF MATHEMATICS

FIN-20014

UNIVERSITY OF TURKU

FINLAND

E-mail address: vuorinen@utu.fi 